\title{
From Persona to Techsona
}

\author{
Susanne Bødker and Clemens Nylandsted Klokmose \\ Department of Computer Science, Aarhus University \\ \{bodker, clemens\}@cs. au. dk
}

\begin{abstract}
In this paper we introduce the notion of techsona, as a possible counterpart design instrument to personas. We use a case study to illustrate a design process where techsonas help pinpoint technological ideas and alternatives and analyze these systematically. While a persona captures "imagine a user...", a scenario "imagine a situation..." the techsona gives the missing piece of "imagine a technology..." We show how a recently developed activity theoretical model can serve both as a framing for techsonas and as an analytical interface between personas and techsonas. The paper discusses the potentials and problems of the techsona and concludes that the techsona truly helps with a missing piece in persona and scenario-based design.
\end{abstract}

Keywords: Persona, Techsona, Activity Theory.

\section{Introduction}

In our work with interaction design, we have used scenarios and personas in various forms over the years. The capacity, and possible and plausible actions of humans are captured and communicated through these. Hence, the use of personas and scenarios help us answer the question of how potential users reach out towards current or hypothetical future technologies. The reverse side, however, needs to be addressed similarly: How do the action possibilities of an artifact, whether a current or hypothetical artifact, reach out towards the potential user? We miss a set of design instruments, matching personas, in order to capture and communicate open issues regarding the artifacts designed, design decisions and even technological alternatives. In this paper we present techsonas to provide this match.

Personas are connected to scenarios in that they often work together, and share the same qualities of capturing both the current situation and human action possibilities on the one hand, and the future on the other [3,5]. Scenarios focus on particular situations, whether typical or critical, and they can generally be used throughout the design process to hold on to design decisions while being open for interpretation. It is our claim in this paper that we need the techsona to counterpart personas and scenarios in order to help users and designers reason about technology on a more abstract and hypothetical level than prototypes. We need means to capture and concretize technological alternatives and thought experiments without necessarily manifesting them as prototypes. Hence, we want to provide a light-weight yet systematic means for addressing questions like: What if we used a wall-mounted multi-touch screen for our ticket vending machine, would that benefit or impede our users? 
Accordingly, we need to populate the gap that we are addressing here with a tool that helps focus on the critical detail in terms of use of future artifacts, and acts as counterparts to personas at the levels from human fear and values to specific action possibilities and constraints. In addition this tool needs to capture elements of existing technologies that are useful when designing the future.

\section{From Personas and Scenarios to Techsonas and Back Again}

Personas $[11,12,13]$ are defined as ways of representing real people throughout the design process. According to Cooper [13], personas are hypothetical archetypes, defined with rigor and precision, discovered in the investigation process. Personas allow for an understanding of the values, fears, etc., of the users [13]. Personas are used in two ways: by their designers in their own design process and for communication with others [17]. In interaction design personas are often used in conjunction with scenarios. Scenarios are most well known from Carroll [9]. He points out that scenarios are stories about people and their activities; that they are specific and fluid at the same time and they point towards both analyses of existing use/work activities and visioning of the future. Making scenarios is a creative process: they are hypotheses, or qualified guesses about the artifact and its use [5]. They serve to open the dialogue about future possibilities and current constraints. $[4,5]$ propose to work from use-like situations towards scenarios that are constructions meant to stage acting in the future or to reflect on and illustrate problems with this action. Our use of personas and scenarios in design is described in [8].

While Carroll [9] argues “...that use-scenarios can be the principle design representation of an artifact” (pp. 190) Bødker \& Christiansen [5] propose to capture and communicate innovative or prototypical examples of technologies, in a manner that seems quite parallel to personas. It is necessary in particular to enable a systematical exploration of new ideas, which is an integral part of any type of interaction design. Hence, we need a means for exploring an (even far-fetched) idea against personas and use scenarios in a lightweight yet systematic manner. Accordingly we see personas and techsonas as closely connected with scenario-based methods, and we have been inspired by previous work on future scenarios [3] and inspiration cards [16].

The difference between personas, techsonas and scenarios can briefly be captured as follows. While a persona in design would easily be approached as "imagine a user...", a scenario as "imagine a situation..." the techsona gives the missing piece of "imagine a technology...". If focusing on a current use situation, personas summarize and crystalize findings regarding users' actions and motives, scenarios flesh out (specific) use situations, and techsonas summarize the action possibilities, or affordances of the technology. Like a persona, the techsona is hypothetical, yet rigorous and precise enough to be confronted with a variety of personas and scenarios.

Where personas are discovered through investigation, techsonas are invented as part of the design process. A good techsona embodies a simple (potential) design idea and provides enough description of its realization to confront the values, fears, capabilities, etc. of the users as expressed in the personas. 
In the following we discuss what goes into a techsona and how can it be confronted with personas in a systematic manner. We use a model derived from activity theory that helps reasoning about the dialectics between human and technology.

\subsection{A Framework for Mediation between Personas and Techsonas}

Recently we have applied the Human-Artifact Model [7] for design-oriented analyses of current and future artifacts and activities of use. In this context the model provides an emphasis on understanding interaction with technology as multi-layered and dialectical. The analytical scheme of the Human-Artifact Model (Figure 1) combines analyses of human experiences and artifacts, and addresses the tensions between human skills and capacity on the one hand, and the action possibilities and affordances offered by the artifact on the other [3]. This is done on three levels reflecting the activity hierarchy: activity, action and operation. These levels provide three sets of analytical glasses: Motivation (by asking why?), goal-orientation (by asking what?) and operation (by asking how?).

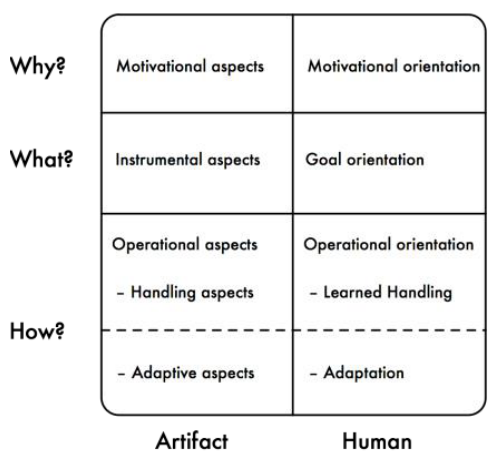

Fig. 1. The Human-Artifact Model (according to [7])

We have combined this approach to understanding the relationship between a practice and given artifacts with iteratively and concurrently developing personas [8]. Through this we confront the richness of personas with the more focused questions of "Why?", "What?" and "How?" coming from the Human-Artifact Model (Figure 1). In the following we illustrate how this is done similarly for the techsona. The model resembles Carroll et al's task-artifact cycle [10. However, while the task-artifact cycle reflects a causal relationship between the artifact and task, the dialectical relationship of the Human-Artifact Model is different: In practical terms, we need to analyze what happens and might happen to the human activity every time artifacts are changed and vice versa, but there no causal predictions available, such as 'because the artifact is changed in this way, the task will change in that'. Another difference between the Human-Artifact Model and the task-artifact model in terms of its focus on tasks, in short, is that tasks are mainly about actions, whereas the why level in particular is missing in the analysis. We refer to [1] for a longer discussion of tasks. 
Applying the Human-Artifact Model as a mediator between personas and techsonas emphasizes their dialectical relationship, and provides a structure for systematically exploring the tensions between the assumptions of the artifact and the capabilities and orientation of the user towards the artifact all the way from motivation to low-level operation.

\section{Case: The Municipal E-service}

The eGov+ project focused on studying and improving eServices for public authorities (see e.g. [6]). The main problem identified were that public authorities often see eServices as replacements of the ways in which citizens show up in the public office to receive e.g. a new passport or a driver's license. The project worked to identify alternative ways of designing and understanding citizen eServices and multiple student projects have been part of this.

Based on records of interviews and observations with citizens and municipal workers, the researchers developed a set of personas that the students were asked to use as starting points when carrying out an iterative design process (see more in [8]). Personas were used to summarize substantial research findings as communication devices within the project, from researchers/analysts to students/designers, similar to the ways personas is described in $[11,15]$. They were supplemented with scenarios to describe the current use situation as well as future use. Here is a condensed description of the persona Ahmed (from [6]):

"Ahmed is a citizen of the world. Yet, somewhat in contrast to this, he is motivated in his life by a wish to own his own home, to live in Denmark. He prefers face-to-face deals in general, and he does e.g. most of his shopping in this manner. He is heavily dominated by his concerns for his close family and their everyday life in Denmark (school, housing, etc.) and his wider family. This family is spread across the world and he makes contact with members whenever possible. Ahmed is used to visit the municipal office in question. He is used to cell phones whereas he does not use Internet banking. He travels with his family to meet relatives whenever possible."

Based on a more detailed description of Ahmed and five other personas, and a selection of scenarios [8] the students were asked to discuss technology suggestions, such as a passport machine coming from a newspaper article, to make rich descriptions of this and their own technological alternatives and relate them to the given personas-these rich descriptions are what we refer to as techsonas. A condensed example the techsona "friend-in-waiting": "The Friend-in-waiting allows citizens who come to the citizen services office to spend the time in the waiting area completing as much passport information as possible. It is used in a similar way as current paper forms, but has the advantages that existing information from municipal systems can be called up automatically. The Friend-in-Waiting is a pad that the front-desk staff hands to people when they enter the office. Hence the Friend-in-waiting does not require for citizens to bring their own device, if only they bring their social security card. The look-and-feel resemblance with paper forms combined with the use of touch 
displays is intended to help citizens who are otherwise not using computer technology. The Friend-in-Waiting is also a guide that leads citizens to a booth where pictures are taken and automatically linked to the ID of the citizen, and, if needed, to the citizen services staff responsible for handling the passport."

The students used The Human-Artifact Model as an interface between personas and techsonas, to analyze potential matches and mismatches between human beings and a technology as it was described, or tensions between persona and techsona. The Human-Artifact Model was also used to structure the development of a techsona, e.g. when the students were asked to develop a techsona based on the passport machine described in a newspaper (Figure 2).

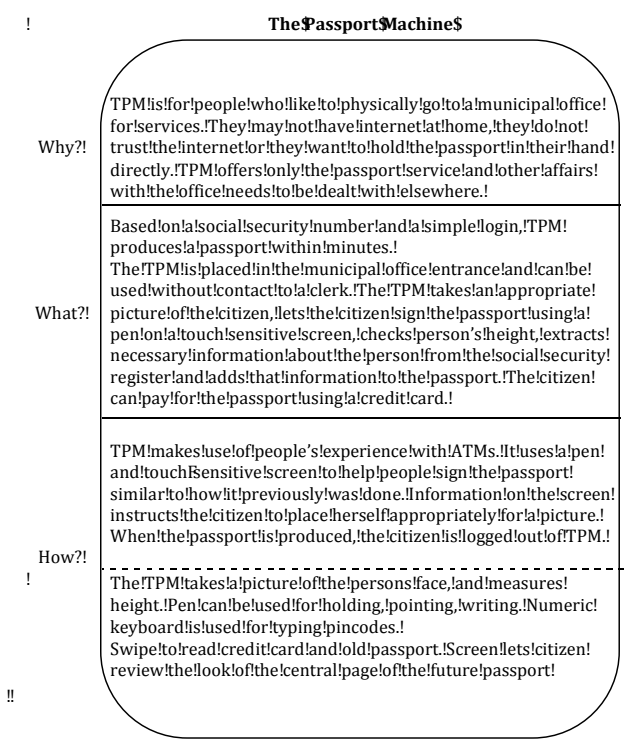

Fig. 2. Creation of techsona through answering the questions of the Human-Artifact Model (The Passport Machine as read about in the newspaper)

From there the students directed their attention towards the future and used the given personas personas to develop scenarios of use, and the Human-Artifact Model to structure their insights regarding the users and use situations. Since the goal was to inform design of a new technological solution used when acquiring a new passport, the personas were coded to focus on e.g. Ahmed's orientation towards a potential new artifact. The Human-Artifact Model supported this coding through its levels: Why would he use the artifact? What will he need to do? How is he used to achieve the goals the artifact realizes? Whether such artifact was useful was debatable, and up for the students to uncover.

Along the way, the students were asked to be skeptic towards their ideas and to provide alternatives. Hence the outcome of the process was four sets of alternatives illustrating four different sets of problems relating to a passport machine. These included the possible use of mobile technology while in the municipal office or away 
from it. One of these alternatives was the 'Friend-in-waiting' - a tablet-based passport device to be used while in the waiting area of citizen services. This helped address a critical issue that had been uncovered in discussions of the passport machine, namely the match of the passport picture with the actual person, which, according to legislation, required physical presence at the office. But how would it help citizens like Ahmed? A techsona was created together with the video-based scenario and coded with the Human-Artifact Model for analysis towards the personas. This techsona was confronted with the personas and new issues occurred that were explored further through traditional means such as a video scenario (exemplified in Figure 3).

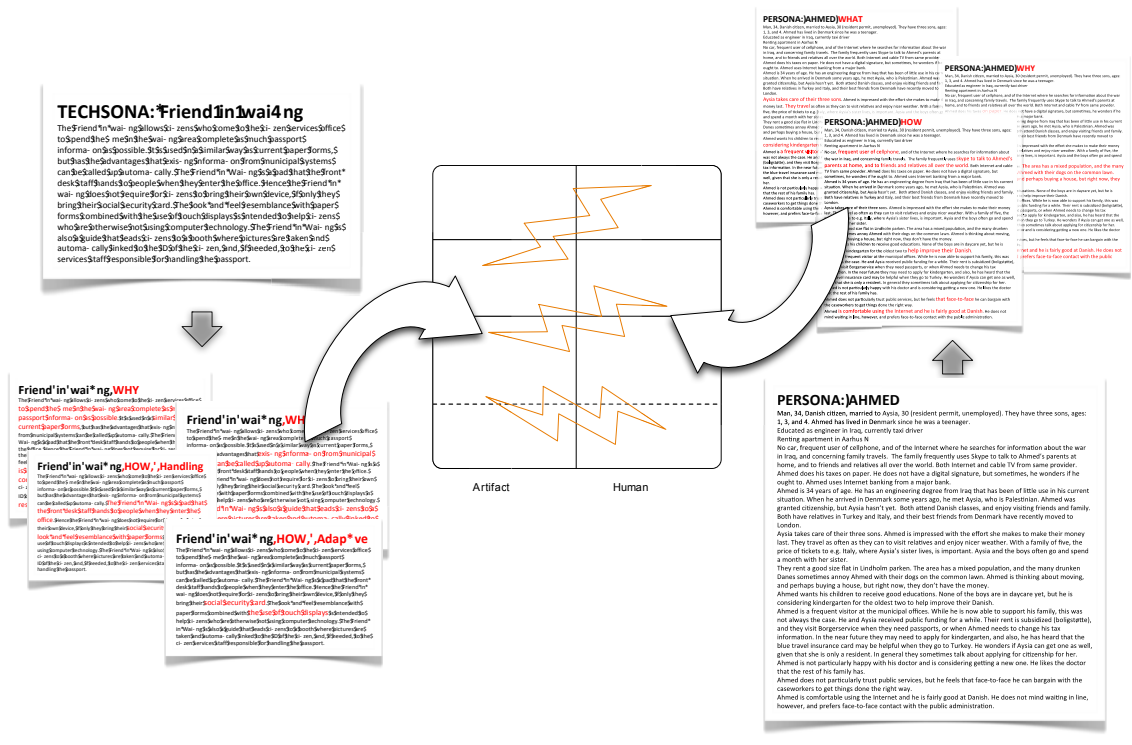

Fig. 3. Human-Artifact Model as a mediator between persona and techsona

\section{The Meeting of Persona and Techsona}

Underlying this work to establish and code the personas and techsonas lies the activity theoretical idea that the human users possess a set of learned and adapted action possibilities, and they are oriented towards certain goals and motives. All of these change throughout the human activity, and they are changeable when meeting and using a new artifact. A new artifact provides new possibilities for use, but it also talks back and cannot be used in any odd way the user may want. Similarly the user cannot necessarily make her capacities work with any new artifact.

Accordingly the meeting of the persona and the techsona is intended to help address the possibilities of a match between the human and the artifact side, as well as the problems. This is not an either/or, rather they are dialectical tensions: Where lie the possibilities that the Persona Ahmed (or one of the other personas) would use the 'Friend-in-Waiting', or the passport machine? What kinds of immediate problems do 
we find that may prevent such future use, or cause a need to adjust the concept before we move on to designing a more elaborate prototype? For instance, would Ahmed's preference for a personal handshake and face-to-face communication prevent him from picking up the 'Friend-in-Waiting'? Or is it sufficient that he meets a person when picking it up and leaving it? Would experience with modern multi-touch interfaces on mobile devices help or hinder his handling of the pad? Or would the idea of picking up a device that is not his own be a barrier? etc. (Figure 3).

The sort of analysis illustrated in this example falls in a tradition that goes back to [4], where the person side and the technology side of activity are systematically confronted with each other through a number of different scenarios, based on systematic questions. The Human-Artifact Model has helped making it more operational in terms of helping students and other designers work in a structured manner with the levels and questions. What we are providing is an analytical tool that helps uncover potentials and problems of future use at an early stage, but obviously, ultimately, there are no firm answers, and this sort of analysis does not prevent problems from occurring in later prototypes or the final design.

\section{Discussion and Conclusion}

In this paper we have introduced techsonas to facilitate a focus on technological possibilities and alternatives in scenario- and persona-based design. We have used the Human-Artifact Model as a mediator between these. The above example illustrates how the analysis brings out critical details, such as in the example, the relationship between experiences with personal devices related to a shared tablet in the case of "friend-in-waiting". While we have limited evidence for this, we suggest that through this kind of analysis we may be able to recognize important details from less important ones, in techsonas, as well as in the personas.

In line with the original motivation for personas, the techsona makes it possible to address motivational issues together with the handling possibilities of the proposed technology. Hence, the techsona targets the role of proposed technology in relation to e.g. human fear and values, and the techsona in that manner is a way of filling the gap that we identified.

There may well be other ways of filling this gap, and we suspect that the HumanArtifact Model structured analysis is best suited for the design of IT, and less so for e.g. a media production where personas similarly are used to represent the target audience. Other theoretical frameworks may serve equally well as a mediator between techsonas on the one hand and scenarios and personas on the other hand. However, the activity theoretical rooting in dialectical thinking is in our opinion crucial to properly understand the relationship between personas and techsonas.

In our experiences with techsonas so far, we have seen indications that this analysis to some extent helps resisting the temptation of premature prototyping, i.e. the temptation to pursue and build a prototypes simply because it looks like a good idea, technically. The students who carefully used the techsonas seemed more critical to their own ideas. Obviously we have built our presentation around one, simplified example. 
Richer material and more cases are behind this presentation, and we are working to gather even more such material. In the meantime, we encourage the readers to start using and developing the ideas further.

Acknowledgements. We thank members of the eGov+ project as well as Karen Johanne Kortbek, Lene Mejlby, Nikolaj G. Borchorst, Marianne Dammand Iversen, Peter Dalsgaard and Kim Halskov for their various contributions. This work has been supported by the Carlsberg Foundation and Aarhus University through the iCenter PIT.

\section{References}

1. Bannon, L., Bødker, S.: Beyond the Interface, Encountering Artifacts in Use. In: Carroll, J. (ed.) Designing Interaction: Psychological Theory of the Human-Computer Interface, pp. 227-253. CUP (1991)

2. Beguin, P.: In search of a unit of analysis for designing instruments. Artifacts 1(1), 12-16 (2007)

3. Bødker, S.: Scenarios - setting the stage for reflection and action in user-centered design. Interacting with Computers 13(1), 61-77 (2000)

4. Bødker, S., Christiansen, E., Thüring, M.: A conceptual toolbox for designing CSCW applications. In: COOP 1995, Juan-les-Pins, pp. 266-284 (January 1995)

5. Bødker, S., Christiansen, E.: Scenarios as springboards in design. In: Bowker, G., Gasser, L., Star, S.L., Turner, W. (eds.) Social Science Research, Technical Systems and Cooperative Work, pp. 217-234. Erlbaum (1997)

6. Bødker, S., Christiansen, E., Nyvang, T., Zander, P.O.: Personas, people and participationchallenges from the trenches of local government. In: 12th Participatory Design Conference, pp. 91-100 (2012)

7. Bødker, S., Klokmose, C.N.: The Human-Artifact Model: An Activity Theoretical Approach to Artifact Ecologies Human-Computer Interaction. HCI Journal, 315-371 (2011)

8. Bødker, S., Klokmose, C.N.: Preparing students for (inter-)action. Journal of Design (2012)

9. Carroll, J.M.: Making Use: Scenario-Based Design of Human-Computer Interactions. MIT Press (2000)

10. Carroll, J.M., Kellogg, W.A., Rosson, M.B.: The Task-Artifact Cycle. In: Carroll, J.M. (ed.) Designing Interaction: Psychology at the Human-Computer Interface. CUP (1991)

11. Chang, Y., Lim, Y., Stolterman, E.: Personas: from theory to practices. In: Nordi-CHI 2005, pp. 439-442. ACM (2008)

12. Cooper, A.: The Inmates are Running the Asylum. SAMS (1999)

13. Friess, E.: Personas and decision making in the design process: An ethnographic study. In: CHI 2012, pp. 1209-1218. ACM (2012)

14. Grudin, J., Pruitt, J.: Personas, participatory design, and product development: An infrastructure for engagement. In: PDC 2002, pp. 144-161 (2002)

15. Halskov, K., Dalsgaard, P.: The emergence of ideas: the interplay between sources of inspiration and emerging design concepts. CoDesign 3(4) (2007)

16. Matthews, T., Judge, T.K., Whittaker, S.: How do designers and user experience professionals actually perceive and use personas? In: CHI 2012, pp. 1219-1228. ACM (2012) 